\title{
GLOBAL STABILIZATION OF SYSTEMS CONTAINING A DOUBLE INTEGRATOR USING A SATURATED LINEAR CONTROLLER
}

\author{
FENG TYAN ${ }^{1}$ AND DENNIS S. BERNSTEIN ${ }^{2, *}$ \\ ${ }^{1}$ Department of Aerospace Engineering, TamKang University, Tamshui, Taipei County, 25137 Taiwan, R.O.C. \\ ${ }^{2}$ Department of Aerospace Engineering, The University of Michigan, Ann Arbor, MI 48109-2140, U.S.A.
}

\begin{abstract}
SUMMARY
In this paper, we present a Lyapunov function for systems containing a double integrator and with controller saturation. This Lyapunov function is composed of a positive-semidefinite quadratic term and an integral term. The main result provides a sufficient condition that guarantees a system with a double integrator can be globally stabilized by a saturating linear controller. For a triple-integrator system the saturated linear controller does not satisfy the sufficient condition, which agrees with the known result. Copyright (C) 1999 John Wiley \& Sons, Ltd.
\end{abstract}

Key words: Lyapunov function; independent saturation; state feedback controller; double integrator

\section{INTRODUCTION}

The input saturation problem is intrinsic to automatic control technology. In fact, no technological advance can circumvent rate and amplitude constraints on electromechanical actuators. Furthermore, cost constraints often force control engineers to extract the best possible performance from components with limited capability, thus increasing the occurrence of saturation. The importance and pervasiveness of saturation is reflected by the extensive research devoted to the problem. See Reference 1 for a recent bibliography.

In practice saturation manifests itself in two ways. First, a control law may command a control input that the actuator is unable to produce either in terms of amplitude or rate. When integral control is used, this situation may entail windup and possible instability. In addition, for chosen control system hardware it is often desirable to extract the best possible performance. In the absence of an energy or fuel constraint, this goal may require that the actuators operate at or near saturation levels in order to maximize performance. In this case actuator operation at saturation

This paper was submitted for publication by editor M. J. Grimble

\footnotetext{
* Correspondence to: Dennis S. Bernstein, Department of Aerospace Engineering, The University of Michigan, Ann Arbor, MI 48109-2140, U.S.A.
}

Contract/grant sponsor: National Science Council, Taiwan

Contract/grant number: NSC-86-2212-E-032-010

CCC 1049-8923/99/151143-14\$17.50

Received 28 June 1999

Copyright (C) 1999 John Wiley \& Sons, Ltd.

Revised 15 July 1999

Accepted 8 August 1999 
limits is not necessarily to be avoided but rather may be desirable so as to maximize the effectiveness of the available control input.

The distinction between stable and unstable plants is important when addressing saturation issues. If the plant is open-loop stable, then saturation is only an issue when performance is quantified since the zero control is unsaturated and stabilizing. On the other hand, global stabilization of plants with open right half-plane poles is impossible in the presence of saturation. In fact, a rare disturbance of high magnitude can perturb the state and render the equilibrium unrecoverable. Therefore, maximizing the domain of attraction for such plants is often the primary objective.

In the context of linear systems with amplitude saturation, there are several fundamental questions concerning the saturation problem that warrant consideration, namely:

1. What is the largest class of systems that can be globally stabilized in the presence of saturation?

2. What is the largest class of systems that can be globally stabilized by a saturated linear controller?

3. For systems that can be globally stabilized by a saturated linear controller, which unsaturated linear globally stabilizing controllers are also globally stabilizing in the presence of saturation?

The first question has been resolved in References 2 and 3, where it was shown that a linear system is globally stabilizable if and only if its poles are contained in the closed left half-plane. Controllers that achieve this objective have been given in References 4 and 5 .

With regard to the second question, it is known that a system with a defective triple (or greater) imaginary axis eigenvalue cannot be globally stabilized by a saturated linear controller. ${ }^{5}$ For systems with a defective double zero eigenvalue, stabilization by saturated linear control is possible, ${ }^{4}$ while the case of a defective double nonzero imaginary eigenvalue is open. Therefore, it remains, in accordance with the third question, to determine which unsaturated linear globally stabilizing controllers are also globally stabilizing in the presence of saturation.

The goal is of this paper is to present a sufficient condition that guarantees closed-loop global stability of saturated linear controllers. Our main result (Theorem 2.1) is a Popov-like sufficient condition based upon a Lure-Posnikov Lyapunov function. ${ }^{6,7}$ This result can be used either to verify global stabilizability of a given saturated linear controller or to synthesize a stabilizing saturated linear controller. Analysis of this sufficient condition shows that it cannot be satisfied when the system contains a triple integrator in accordance with the results of Reference 4.

\section{ANALYSIS OF SYSTEMS WITH SATURATION NONLINEARITY}

We consider the closed-loop system

$$
\begin{aligned}
& \dot{x}(t)=A x(t)+B \sigma(u(t)), \quad x(0)=x_{0} \\
& u(t)=K x(t)
\end{aligned}
$$

where

$$
x \triangleq\left[\begin{array}{l}
x_{z} \\
x_{s}
\end{array}\right] \in \mathbb{R}^{n}, \quad A \triangleq\left[\begin{array}{cc}
A_{z} & 0 \\
0 & A_{s}
\end{array}\right], \quad B \triangleq\left[\begin{array}{c}
B_{z} \\
B_{s}
\end{array}\right], \quad K \triangleq\left[\begin{array}{ll}
K_{z} & K_{s}
\end{array}\right]
$$


in which $x_{z} \in \mathbb{R}^{n_{z}}, x_{s} \in \mathbb{R}^{n_{s}}, n=n_{z}+n_{s}, u \in \mathbb{R}^{m}$ is the control input, and $A_{z}, A_{s}, B_{z}, B_{s}, K_{z}, K_{s}$, are real matrices. We assume that $\operatorname{spec}\left(A_{z}\right) \subset \mathrm{IA}$ and $\operatorname{spec}\left(A_{s}\right) \subset \mathrm{OLHP}$. The function $\sigma: \mathbb{R}^{m} \rightarrow \mathbb{R}^{m}$ is an independent symmetric saturation function, that is, $\sigma(u) \triangleq\left[\sigma_{1}\left(u_{1}\right) \cdots \sigma_{m}\left(u_{m}\right)\right]^{\mathrm{T}}$, where

$$
\sigma_{i}\left(u_{i}\right) \triangleq \operatorname{sat}_{\tilde{u}_{i}}\left(u_{i}\right), \quad i=1, \ldots, m
$$

$\tilde{u}_{i}>0$ is the saturation level of $\sigma_{i}$, and, for $\alpha>0$,

$$
\begin{array}{rlrl}
\operatorname{sat}_{\alpha}(v) & =v, & & |v| \leqslant \alpha \\
& =\operatorname{sgn}(v) \alpha, & |v|>\alpha
\end{array}
$$

For $m \geqslant 2$ the saturation function $\sigma(\cdot)$ may change the direction of the control input, that is, $\sigma(u(t))$ is not necessarily in the same direction as $u(t)$. Note that $\sigma(u)$ can be written as

$$
\sigma(u)=\beta(u) u
$$

where $\beta(u) \triangleq \operatorname{diag}\left(\beta_{1}\left(u_{1}\right), \ldots, \beta_{m}\left(u_{m}\right)\right)$ and $\beta_{i}: \mathbb{R} \rightarrow(0,1], i=1, \ldots, m$, is defined by

$$
\begin{aligned}
\beta_{i}\left(u_{i}\right) & =1, & \left|u_{i}\right| \leqslant \bar{u}_{i} \\
& =\frac{\bar{u}_{i}}{\left|u_{i}\right|}, & \left|u_{i}\right|>\bar{u}_{i}
\end{aligned}
$$

Our main result is the following.

\section{Theorem 2.1}

Let

$$
\begin{gathered}
R_{z} \in \mathbb{N}^{n_{z} \times n_{z}}, \quad R_{s} \in \mathbb{N}^{n_{s} \times n_{s}} \quad R \triangleq\left[\begin{array}{cc}
R_{z} & 0 \\
0 & R_{s}
\end{array}\right], \quad R_{2} \in \mathbb{D N}^{m \times m}, N=\operatorname{diag}\left(N_{1}, \ldots, N_{m}\right) \in \mathbb{D N}^{m \times m}, \\
K=\left[\begin{array}{c}
K_{1} \\
\vdots \\
K_{m}
\end{array}\right], \quad K_{i} \in \mathbb{R}^{1 \times\left(n_{z}+n_{s}\right)}, \quad i=1, \ldots, m
\end{gathered}
$$

and assume that either (i) $(A, K)$ is observable, or (ii) $\left(A_{s}, R_{s}\right)$ is observable and $(A, K)$ is detectable. Furthermore, suppose there exists $P \in \mathbb{N}^{n \times n}$ satisfying

$$
\begin{aligned}
& 0=A^{\mathrm{T}} P+P A+R \\
& 0=B^{\mathrm{T}} P+N K A+R_{2} K \\
& 0<2 R_{2}-\left(N K B+B^{\mathrm{T}} K^{\mathrm{T}} N\right) \\
& 0<P+K^{\mathrm{T}} N K
\end{aligned}
$$

Then the closed-loop system (1) and (2) is globally asymptotically stable with the Lyapunov function

$$
V(x)=x^{\mathrm{T}} P x+2 \sum_{i=1}^{m} \int_{0}^{K_{x} x} N_{i} \sigma_{i}\left(u_{i}\right) \mathrm{d} u_{i}
$$


Furthermore, the cost functional

$$
J\left(x_{0}\right)=\int_{0}^{\infty}\left[x^{\mathrm{T}}(t) R x(t)+2 u^{\mathrm{T}}(t) R_{2} \sigma(u(t))-\sigma^{\mathrm{T}}(u(t))\left(N K B+B^{\mathrm{T}} K^{\mathrm{T}} N\right) \sigma(u(t))\right] \mathrm{d} t
$$

satisfies $J\left(x_{0}\right)=V\left(x_{0}\right)$.

Proof. To show that $V(x)$ is positive definite, note that

$$
\begin{aligned}
V^{-1}(0) & =\left\{x \in \mathbb{R}^{n}: x^{\mathrm{T}} P x=0\right\} \cap\left\{x \in \mathbb{R}^{n}: N_{1} K_{1} x=0\right\} \cap \cdots \cap\left\{x \in \mathbb{R}^{n}: N_{m} K_{m} x=0\right\} \\
& =\left\{x \in \mathbb{R}^{n}: x^{\mathrm{T}} P x=0\right\} \cap\left\{x \in \mathbb{R}^{n}: N K x=0\right\} \\
& =\left\{x \in \mathbb{R}^{n}: x^{\mathrm{T}}\left(P+K^{\mathrm{T}} N K\right) x=0\right\}
\end{aligned}
$$

It then follows from (7) that $V^{-1}(0)=\{0\}$. Hence, $V$ is positive definite. Next, by using (1) and (2), the derivative $\dot{V}(x)$ of $V(x)$ along a trajectory of the closed-loop system can be written as

$$
\dot{V}(x)=\left[\begin{array}{ll}
x^{\mathrm{T}} & \sigma^{\mathrm{T}}(u)
\end{array}\right]\left[\begin{array}{cc}
A^{\mathrm{T}} P+P A & P B+A^{\mathrm{T}} K^{\mathrm{T}} N \\
B^{\mathrm{T}} P+N K A & N K B+B^{\mathrm{T}} K^{\mathrm{T}} N
\end{array}\right]\left[\begin{array}{c}
x \\
\sigma(u)
\end{array}\right]
$$

Adding and subtracting $2 \sigma^{\mathrm{T}} R_{2}[K x-\sigma(u)]$ and using (4) and (5) yields

$$
\begin{aligned}
\dot{V}(x)= & -x^{\mathrm{T}} R x-2 \sigma^{\mathrm{T}} R_{2}[K x-\sigma(u)]-\sigma^{\mathrm{T}}\left[2 R_{2}-\left(N K B+B^{\mathrm{T}} K N\right)\right] \sigma(u) \\
= & -x^{\mathrm{T}} R x-2 u^{\mathrm{T}} \beta(u) R_{2}[I-\beta(u)] u \\
& -x^{\mathrm{T}} K^{\mathrm{T}} \beta(u)\left[2 R_{2}-\left(N K B+B^{\mathrm{T}} K N\right)\right] \beta(u) K x \\
= & -x_{z}^{\mathrm{T}} R_{z} x_{z}-x_{s}^{\mathrm{T}} R_{s} x_{s}-2 u^{\mathrm{T}} \beta(u) R_{2}[I-\beta(u)] u \\
& -\left[x_{s}^{\mathrm{T}} K_{s}^{\mathrm{T}}+x_{z}^{\mathrm{T}} K_{z}^{\mathrm{T}}\right] \beta(u)\left[2 R_{2}-\left(N K B+B^{\mathrm{T}} K N\right)\right] \beta(u)\left[K_{s} x_{s}+K_{z} x_{z}\right] \\
\leqslant & 0 .
\end{aligned}
$$

Thus $\dot{V}(x)=0$ implies that $R_{z} x_{z}=0, R_{s} x_{s}=0$ and $u=K x=K_{s} x_{s}+K_{z} x_{z}=0$. In this case, the system dynamics becomes $\dot{x}(t)=A x(t)$. In addition, if $(A, K)$ is observable, then it follows that the column vectors of $K \mathrm{e}^{A t}$ are linearly independent. Hence the set $x \in \mathbb{R}^{n}$ such that $\dot{V}(x)=0$ consists of $x=0$ only. Similarly, the observability of $\left(A_{s}, R_{s}\right)$ implies that $x_{s}=0$, and the detectability of $(A, K)$ is equivalent to the observability of $\left(A_{z}, K_{z}\right)$, which in turn implies that $x_{z}=0$. Hence in both cases the invariant set of $\dot{V}(x)=0$ consists of 0 . It follows that $V(x(t)) \rightarrow 0$ as $t \rightarrow \infty$ and the closed-loop system (1), (2) is globally asymptotically stable. Finally,

$$
\begin{aligned}
J\left(x_{0}\right) & =\int_{0}^{\infty}\left[x^{\mathrm{T}}(t) R x(t)+2 u^{\mathrm{T}}(t) R_{2} \sigma(u(t))-\sigma^{\mathrm{T}}(u(t))\left(N K B+B^{\mathrm{T}} K^{\mathrm{T}} N\right) \sigma(u(t))\right] \mathrm{d} t \\
& =\int_{0}^{\infty}-\dot{V}(x(t)) \mathrm{d} t \\
& =V(x(0)) .
\end{aligned}
$$

\section{Remark 2.1}

Unlike Reference 7 note that $P \geqslant 0$ and $R_{2} \geqslant 0$ instead of $P>0$ and $R_{2}>0$. 
Remark 2.2

Forming (4) $+K^{\mathrm{T}}(5)+(5)^{\mathrm{T}} K$, it follows that

$0=(A+B K)^{\mathrm{T}}\left(P+K^{\mathrm{T}} N K\right)+\left(P+K^{\mathrm{T}} N K\right)(A+B K)+R+K^{\mathrm{T}}\left(2 R_{2}-B^{\mathrm{T}} K^{\mathrm{T}} N-N K B\right) K$.

Furthermore if (6) is satisfied and $(A, K)$ is detectable then it follows from (7) that $A+B K$ is asymptotically stable.

Remark 2.3

Theorem 2.1 can be used to guarantee global stability of a given controller, or it can be used to construct a saturated linear controller.

\section{DOUBLE INTEGRATOR}

To shed the light on the structure of solutions when $n_{z} \geqslant 1$, partition

$$
P \triangleq\left[\begin{array}{cc}
P_{z} & P_{z s} \\
P_{z s}^{\mathrm{T}} & P_{s}
\end{array}\right]
$$

and write (4)-(7) as

$$
\begin{aligned}
0 & =\left[\begin{array}{cc}
A_{z}^{\mathrm{T}} P_{z}+P_{z} A_{z}+R_{z} & A_{z}^{\mathrm{T}} P_{z s}+P_{z s} A_{s} \\
A_{s}^{\mathrm{T}} P_{z s}^{\mathrm{T}}+P_{z s}^{\mathrm{T}} A_{z} & A_{s}^{\mathrm{T}} P_{s}+P_{s} A_{s}+R_{s}
\end{array}\right] \\
0 & =\left[\begin{array}{ll}
B_{z}^{\mathrm{T}} P_{z}+N K_{z} A_{z}+R_{2} K_{z} & B_{s}^{\mathrm{T}} P_{s}+N K_{s} A_{s}+R_{2} K_{s}
\end{array}\right] \\
0 & <2 R_{2}-\left(N K_{z} B_{z}+B_{z}^{\mathrm{T}} K_{z}^{\mathrm{T}} N+N K_{s} B_{s}+B_{s}^{\mathrm{T}} K_{s}^{\mathrm{T}} N\right) \\
0 & <\left[\begin{array}{cc}
P_{z}+K_{z}^{\mathrm{T}} N K_{z} & P_{z s}+K_{z}^{\mathrm{T}} N K_{s} \\
P_{z s}^{\mathrm{T}}+K_{z}^{\mathrm{T}} N K_{z} & P_{s}+K_{s}^{\mathrm{T}} N K_{s}
\end{array}\right]
\end{aligned}
$$

The following technical lemma is critical to our development.

\section{Lemma 3.1}

Let $R_{z} \in \mathbb{N}^{n_{z} \times n_{z}}$. Then there exists $P_{z} \in \mathbb{N}^{n_{z} \times n_{z}}$ satisfying

$$
0=A_{z}^{\mathrm{T}} P_{z}+P_{z} A_{z}+R_{z}
$$

if and only if $R_{z}=0$.

Proof. If $R_{z}=0$ then choose $P_{z}=0$. Conversely, we transform $A_{z}$ into real Jordan form $A_{z}=\operatorname{diag}\left(A_{z \omega_{0}}, A_{z \omega_{1}}, \ldots, A_{z \omega_{f}}\right)$, where $A_{z \omega_{i}}=\operatorname{diag}\left(A_{z \omega_{i}(1,1)}, \ldots, A_{z \omega_{i}\left(r_{i}, r_{i}\right)}\right), i=0,1, \ldots, f$, and $A_{z \omega_{i}(j, j)}$ has the structure

$$
\begin{aligned}
& A_{z \omega_{i}(j, j)} \\
& =0 \text { or } Z_{2} \text { or } Z_{3} \cdots, i=0, j=1, \ldots, r_{0} \\
& =\omega_{i} J \text { or }\left(\omega_{i} I_{2} \otimes J+Z_{2} \otimes I_{2}\right) \text { or }\left(\omega_{i} I_{3} \otimes J+Z_{3} \otimes I_{2}\right) \cdots, i=1, \ldots, f, j=1, \ldots, r_{i}
\end{aligned}
$$


where $\omega_{i}>0$. Then it is easy to show that there exists a positive semidefinite solution $P_{z \omega_{i}(j, j)}$ for the diagonal terms of equation (9), i.e.,

$$
0=A_{z \omega_{i}(j, j)}^{\mathrm{T}} P_{z \omega_{i}(j, j)}+P_{z \omega_{i}(j, j)} A_{z \omega_{i}(j, j)}+R_{z \omega_{i}(j, j)}, i=0,1, \ldots, f, j=1, \ldots, r(i)
$$

if and only if $R_{c \omega_{i}(j, j)}=0, i=0,1, \ldots, f, j=1, \ldots, r_{i}$. Indeed for each $A_{z \omega_{i}(j, j)}$ its corresponding solution $P_{z \omega_{i}(j, j)}$ is in the form of

$$
\begin{aligned}
P_{z \omega_{i}(j, j)} & =p_{z i j} \text { or }\left[\begin{array}{cc}
0 & 0 \\
0 & p_{z i j}
\end{array}\right] \text { or }\left[\begin{array}{ccc}
0 & 0 & 0 \\
0 & 0 & 0 \\
0 & 0 & p_{z i j}
\end{array}\right] \ldots, i=0, j=1, \ldots, r_{0} \\
& =p_{z i j} I_{2} \text { or }\left[\begin{array}{cc}
0 & 0 \\
0 & p_{z i j} I_{2}
\end{array}\right] \text { or }\left[\begin{array}{ccc}
0 & 0 & 0 \\
0 & 0 & 0 \\
0 & 0 & p_{z i j} I_{2}
\end{array}\right] \ldots, i=1, \ldots, f, j=1, \ldots, r_{i}
\end{aligned}
$$

where $p_{z i j}>0, i=0,1, \ldots, f, j=1, \ldots, r_{i}$. However, since $R_{z}$ is assumed to be positive semidefinite, it follows that $R_{z}=0$. Note that in this case $P_{z}=\operatorname{diag}\left(P_{z \omega_{0}} P_{z \omega_{1}} \cdots P_{z \omega_{f}}\right)$, where $P_{z \omega_{i}}=\operatorname{diag}\left(P_{z \omega_{i}(1,1)} \cdots P_{z \omega_{i}(r(i), r(i))}\right), i=0,1, \ldots, f$.

\section{Remark 3.1}

Lemma 3.1 can be read as follows: Let $P_{z}$ be non-negative definite and let $A_{z}$ be such that all of its eigenvalues have zero real part. Then the matrix $A_{z}^{\mathrm{T}} P_{z}+P_{z} A_{z}$ is either zero or it has at least one positive and one negative eigenvalue.

\section{Lemma 3.2}

Assume that $\left(A_{z}, B_{z}\right)$ is controllable. Then there exists $P_{z} \in \mathbb{N}^{n_{z} \times n_{z}}, R_{2} \in \mathbb{D} \mathbb{N}^{m \times m}, N \in \mathbb{D N}^{m \times m}$, and $K_{z} \in \mathbb{R}^{n_{z} \times m}$ such that rank $\left(\left[N R_{2}\right]\right)=m$ and

$$
\begin{aligned}
& 0=A_{z}^{\mathrm{T}} P_{z}+P_{z} A_{z} \\
& 0=B_{z}^{\mathrm{T}} P_{z}+N K_{z} A_{z}+R_{2} K_{z} \\
& 0<P_{z}+K_{z}^{\mathrm{T}} N K_{z}
\end{aligned}
$$

if and only if every eigenvalue of $A_{z}$ is semisimple, except zero which may appear as a defective double eigenvalue. Furthermore, in this case $\left(A_{z}, K_{z}\right)$ is observable.

Proof. In order not to be overwhelmed by notation, we can simply assume that $A_{z}$ contains one subsystem, and in this subsystem there is only one Jordan block. We also assume that $\left(A_{z}, B_{z}\right)$ is in controllable canonical form. We start by proving the sufficiency part, that is, $A_{z}$ contains simple poles on the $j \omega$ axis or double poles at the origin. It is easy to see that if $\left(A_{z}, B_{z}\right)=([0],[1])$, then $\left(P_{z}, K_{z}, N, R_{2}\right)=(0, k, 1,0)$ or $(-k, k, 0,1)$ satisfies (12)-(14), where $k<0$. If

$$
\left(A_{z}, B_{z}\right)=\left(\left[\begin{array}{ll}
0 & 1 \\
0 & 0
\end{array}\right],\left[\begin{array}{c}
b_{1} \\
1
\end{array}\right]\right)
$$


where $b_{1}$ is arbitrary, then

$$
\left(P_{z}, K_{z}, N, R_{2}\right)=\left(\left[\begin{array}{cc}
0 & 0 \\
0 & -k_{1}
\end{array}\right], \quad\left[\begin{array}{ll}
k_{1} & k_{2}
\end{array}\right], \quad 1,0\right)
$$

satisfies (12)-(14) where $k_{1}<0$ and $k_{2}$ is arbitrary. If $A_{z}=\omega_{1} J$, where $\omega_{1}>0$, and $\left(A_{z}, B_{z}\right)$ is controllable but otherwise $B_{z}$ is arbitrary, then $\left(P_{z}, K_{z}, N, R_{2}\right)=\left(p_{z 11} I_{2},-p_{z 11} R_{2}^{-1} B_{z}^{\mathrm{T}}, 0, R_{2}\right)$ satisfy (12)-(14), where $p_{z 11}>0, R_{2}>0$. We prove the necessity part by contradiction. First consider a triple pole at the origin so that

$$
A_{z}=\left[\begin{array}{lll}
0 & 1 & 0 \\
0 & 0 & 1 \\
0 & 0 & 0
\end{array}\right]
$$

Then

$$
P_{z}=\left[\begin{array}{ccc}
0 & 0 & 0 \\
0 & 0 & 0 \\
0 & 0 & p_{c 0}
\end{array}\right]
$$

where $p_{c 0}>0$, and it can be seen that, for all values of $B_{z}$, equation (14) cannot be satisfied, and hence the solutions of (12)-(14) do not exist. Next, for non-zero double poles on the j $\omega$ axis,

$$
A_{z}=\left[\begin{array}{cc}
\omega_{1} J & I_{2} \\
0 & \omega_{1} J
\end{array}\right]
$$

where $\omega_{1}>0$, it follows that

$$
P_{z}=\left[\begin{array}{cc}
0 & 0 \\
0 & p_{z 11} I_{2}
\end{array}\right]
$$

where $p_{z 11}>0$. Again it is easy to see that, for all values of $B_{z},(14)$ cannot be satisfied, and hence the solutions do not exist. Similarly, for three or more poles located at the same place on the $j \omega$ axis, it can be shown that solutions of (12)-(14) do not exist. Through the structure of the solution $K_{z}$, it can be seen from the PBH test that $\left(A_{z}, K_{z}\right)$ is observable. This completes the proof.

\section{Remark 3.2}

The proof of Lemma 3.2 suggests that a non-zero defective double pole on the $j \omega$ axis cannot be globally stabilized by using a saturated linear state feedback controller. However, this problem remains open.

From the above lemmas, we have the following result which provides a necessary condition for the existence of a solution to (4)-(7).

\section{Theorem 3.1}

Suppose that $P$ and $R$ satisfy (4)-(7). Then $P$ and $R$ are of the form

$$
P=\left[\begin{array}{cc}
P_{z} & 0 \\
0 & P_{z}
\end{array}\right], \quad R=\left[\begin{array}{cc}
0 & 0 \\
0 & R_{s}
\end{array}\right]
$$


where $P_{z} \in \mathbb{N}^{n_{z} \times n_{z}}, P_{s} \in \mathbb{N}^{n_{s} \times n_{s}}, R_{s} \in \mathbb{N}^{n_{s} \times n_{s}}$, and every eigenvalue of $A_{z}$ is semisimple, except the one at the origin which can be a double defective eigenvalue.

\section{EXAMPLES}

\section{Example 4.1}

Consider the second-order open-loop asymptotically or Lyapunov stable system

$$
\begin{aligned}
& \dot{x}(t)=\left[\begin{array}{cc}
0 & 1 \\
\alpha_{1} & \alpha_{2}
\end{array}\right] x(t)+\left[\begin{array}{l}
0 \\
1
\end{array}\right] \sigma(u(t)) \\
& u(t)=\left[\begin{array}{ll}
k_{1} & k_{2}
\end{array}\right] x(t)
\end{aligned}
$$

where $\alpha_{1}<0, \alpha_{2} \leqslant 0, k_{1}<0, k_{2}<0$. Letting

$$
N=1, R=\left[\begin{array}{cc}
r_{1} & 0 \\
0 & r_{2}
\end{array}\right]
$$

where

$$
\begin{aligned}
& r_{1}= \begin{cases}2 \alpha_{1}\left(\alpha_{1} K_{2}+R_{2} K_{1}\right) & \text { if } \alpha_{2} \neq 0, \\
0 & \text { if } \alpha_{2}=0\end{cases} \\
& r_{2}= \begin{cases}2 \alpha_{2}\left(\frac{r_{1}}{2 \alpha_{1} \alpha_{2}}+R_{2} K_{2}+K_{1}+\alpha_{2} K_{2}\right) & \text { if } \alpha_{2} \neq 0, \\
0 & \text { if } \alpha_{2}=0\end{cases}
\end{aligned}
$$

and $R_{2}$ satisfies

$$
\begin{array}{ll}
\left(-\frac{\alpha_{1} K_{2}}{K_{1}}<R_{2}\right) \text { and }\left(-\alpha_{2}-\frac{\alpha_{1} K_{2}}{K_{1}+\alpha_{2} K_{2}}<R_{2}\right) & \text { if } \alpha_{2} \neq 0 \text { and } K_{1}+\alpha_{2} K_{2}>0 \\
-\frac{\alpha_{1} K_{2}}{K_{1}}<R_{2}<-\alpha_{2}-\frac{\alpha_{1} K_{2}}{K_{1}+\alpha_{2} K_{2}} & \text { if } \alpha_{2} \neq 0 \text { and } K_{1}+\alpha_{2} K_{2}<0 \\
R_{2}>-\frac{\alpha_{1} K_{2}}{K_{1}} & \text { if } \alpha_{2} \neq 0 \text { and } K_{1}+\alpha_{2} K_{2}=0 \\
R_{2}=-\frac{\alpha_{1} K_{2}}{K_{1}} & \text { if } \alpha_{2}=0
\end{array}
$$

it can be shown that (4)-(7) are satisfied. In addition, we have the required conditions that either (i) $(A, R)$ is observable and $(A, K)$ is detectable (when $\alpha_{2} \neq 0$ ), or (ii) $(A, K)$ is observable (when $\alpha_{2}=0$ ). Hence, Theorem 2.1 implies that $x=0$ is a globally asymptotically stable equilibrium point of the saturated closed-loop system for every saturation level $\bar{u}_{1}>0$. 


\section{Example 4.2}

Consider the open-loop asymptotically stable system

$$
\dot{x}(t)=A x(t)+B \sigma(K x(t))
$$

where $A \in \mathbb{R}^{n \times n}, B \in \mathbb{R}^{n \times m}, K \in \mathbb{R}^{m \times n}$, and $(A, B)$ is controllable. Consider LQR design with

$$
J=\int_{0}^{\infty}\left[x^{\mathrm{T}}(t)\left(R+P B R_{2}^{-1} B^{\mathrm{T}} P\right) x(t)+u^{\mathrm{T}}(t) R_{2} u(t)\right] \mathrm{d} t
$$

where $R \in \mathbb{P}^{m \times m}, R_{2} \in \mathbb{P}^{m \times m}$, and $R_{2}$ is diagonal, $P \in \mathbb{P}^{n \times n}$, satisfies $A^{\mathrm{T}} P+P A+R=0$ and $K=-R_{2}^{-1} B^{\mathrm{T}} P$. Note that $(A, R)$ is observable. Then it is straightforward to see that (4)-(7) are satisfied with $N=0$. Hence the resulting closed-loop system is globally asymptotically stable.

\section{Example 4.3}

Consider system (15) where $A \in \mathbb{R}^{n \times n}, B \in \mathbb{R}^{n \times m}, K \in \mathbb{R}^{m \times n},(A, B)$ is controllable and $A$ is skew symmetric. Consider LQR design with

$$
J=\int_{0}^{\infty}\left[x^{\mathrm{T}}(t) B R_{2}^{-1} B^{\mathrm{T}} x(t)+u^{\mathrm{T}}(t) R_{2} u(t)\right] \mathrm{d} t
$$

where $R_{2} \in \mathbb{P}^{m \times m}$ is diagonal, and $K=-R_{2}^{-1} B^{\mathrm{T}}$. The PBH test and the controllability of $(A, B)$ imply

$$
\operatorname{rank}\left[\begin{array}{c}
\lambda I-A \\
K
\end{array}\right]=\operatorname{rank}\left[\begin{array}{c}
\lambda I+A^{\mathrm{T}} \\
-R_{2}^{-1} B^{\mathrm{T}}
\end{array}\right]=\operatorname{rank}\left[\lambda I+A-B R_{2}^{-1}\right]=n, \lambda \in \mathbb{C}
$$

which implies that $(A, K)$ is observable. Then it is straightforward to see that (4)-(7) are satisfied with $P=I_{n}$, and $N=0$. Hence the resulting closed-loop system is globally asymptotically stable.

\section{Example 4.4}

Consider the double integrator plant (4.1) where

$$
A=\left[\begin{array}{ll}
0 & 1 \\
0 & 0
\end{array}\right], \quad B=\left[\begin{array}{l}
0 \\
1
\end{array}\right], \quad x=\left[\begin{array}{l}
x_{1} \\
x_{2}
\end{array}\right], \quad K=\left[\begin{array}{ll}
k_{1} & k_{2}
\end{array}\right]
$$

Let $N=1, R_{2}=0, R=0$ and consider the Lyapunov function

$$
V(x)=x^{\mathrm{T}} P x+2 \int_{0}^{K x} \sigma(u) \mathrm{d} u
$$

where $P \in \mathbb{N}^{2 \times 2}$. Solving (4) and (5) gives

$$
P=\left[\begin{array}{cc}
0 & 0 \\
0 & -k_{1}
\end{array}\right]
$$

Inequalities (6) and (7) then require $k_{2}<0$ and $k_{1}<0$, respectively. This shows that for arbitrary $k_{1}<0, k_{2}<0$, the origin of the corresponding closed-loop system with $u=k_{1} x_{1}+k_{2} x_{2}$ is globally asymptotically stable. Note that in this example, $R_{2}$ must be 0 since otherwise inequality 


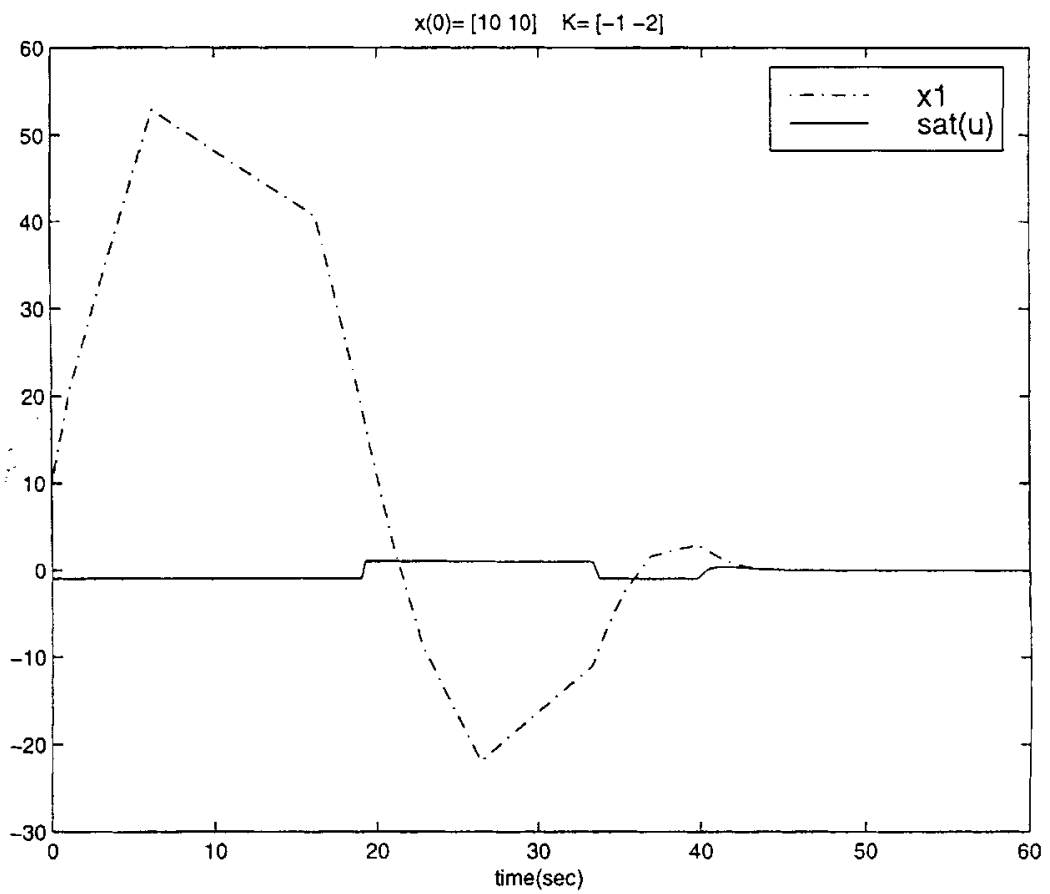

Figure 1. Response $x_{1}(t)$ of the system given in Example 4.4

(7) cannot be satisfied. For illustration purpose, Figure 1 shows the system response $x_{1}(t)$ with initial condition $x(0)=\left[\begin{array}{ll}10 & 10\end{array}\right]^{\mathrm{T}}$, controller gain $K=\left[\begin{array}{ll}-1 & -2\end{array}\right]$ and saturation level $\bar{u}_{1}=1$.

\section{Example 4.5}

Consider a system, taken from Reference 11, containing a double integrator,

$$
\begin{aligned}
& \dot{x}(t)=\left[\begin{array}{rrrr}
0 & 1 & 0 & 0 \\
0 & 0 & 1 & 0 \\
0 & 0 & 0 & 1 \\
0 & 0 & -2 & -2
\end{array}\right] x(t)+\left[\begin{array}{l}
0 \\
0 \\
0 \\
1
\end{array}\right] u(t), \quad x(0)=\left[\begin{array}{r}
0 \\
0 \\
10 \\
-15
\end{array}\right] \\
& u(t)=\operatorname{sat}(K x(t))
\end{aligned}
$$

\section{Choose}

$$
R=\left[\begin{array}{llll}
0 & 0 & 0 & 0 \\
0 & 0 & 0 & 0 \\
0 & 0 & 1 & 0 \\
0 & 0 & 0 & 1
\end{array}\right], \quad R_{2}=0 \text { and } N=1
$$


The solution $P$ of (4) has the form

$$
P=\left[\begin{array}{cccc}
0 & 0 & 0 & 0 \\
0 & p & p & \frac{1}{2} p \\
0 & p & p+\frac{5}{4} & \frac{1}{2} p+\frac{1}{4} \\
0 & \frac{1}{2} p & \frac{1}{2} p+\frac{1}{4} & \frac{1}{4} p+\frac{3}{8}
\end{array}\right]
$$

where $p \geqslant 0$. The gain $K \triangleq\left[\begin{array}{llll}k_{1} & k_{2} & k_{3} & k_{4}\end{array}\right]$ is required to satisfy equation (5), which is equivalent to

$$
0=\left[\begin{array}{llll}
0 & \frac{1}{2} p & \frac{1}{2} p+\frac{1}{4} & \frac{1}{4} p+\frac{3}{8}
\end{array}\right]+\left[\begin{array}{lllll}
0 & k_{1} & k_{2}-2 k_{4} & k_{3}-2 k_{4}
\end{array}\right]
$$

Hence

$$
K=\left[\begin{array}{llll}
-\frac{1}{2} p & 2 k_{4}-\frac{1}{2} p-\frac{1}{4} \quad 2 k_{4}-\frac{1}{4} p-\frac{3}{8} \quad k_{4}
\end{array}\right]
$$

To satisfy (6), $k_{4}<0$ is required. Finally, since $\operatorname{det}\left(P+K^{\mathrm{T}} N K\right)=\frac{13}{128} p^{3}$, we have $P+K^{\mathrm{T}} N K>0$ if $p>0$. This shows the state feedback controller $u(t)=\operatorname{sat}(K x(t))$ is globally stabilizing with $K$ given by (16), where $p>0$ and $k_{4}<0$. For illustration purpose, Figure 2 shows the system response with $p=2, k_{4}=-1$ and saturation level $\bar{u}_{1}=1$.

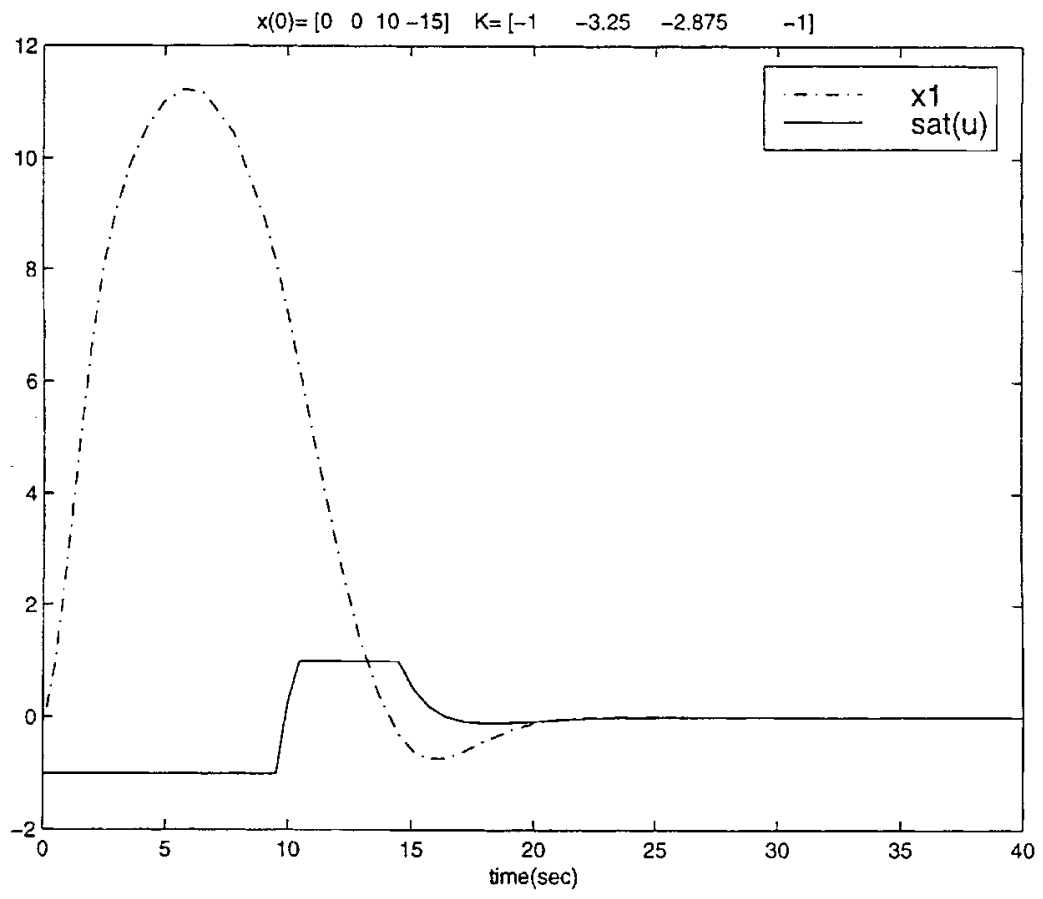

Figure 2. Response $x_{1}(t)$ of the system given in Example 4.5 


\section{Example 4.6}

Although Theorem 2.1 is a sufficient condition, the following example illustrates that a triple integrator cannot be stabilized globally by means of a saturated linear controller which agrees with the known result proved also by Fuller. ${ }^{8}$ Consider the triple integrator system (16) where

$$
A=\left[\begin{array}{lll}
0 & 1 & 0 \\
0 & 0 & 1 \\
0 & 0 & 0
\end{array}\right], \quad B=\left[\begin{array}{l}
0 \\
0 \\
1
\end{array}\right]
$$

and $K \in \mathbb{R}^{1 \times 3}$. Again, by letting $R=0, N=1$ equations (4)-(6) can be written as

$$
\begin{aligned}
0 & =\left[\begin{array}{ccc}
0 & p_{11} & p_{12} \\
p_{11} & 2 p_{12} & p_{13}+p_{22} \\
p_{12} & p_{13}+p_{22} & 2 p_{23}
\end{array}\right] \\
0 & =\left[\begin{array}{lll}
p_{13}+R_{2} k_{1} & p_{23}+k_{1}+R_{2} k_{2} & p_{33}+k_{2}+R_{2} k_{3}
\end{array}\right] \\
0 & <R_{2}-k_{3}
\end{aligned}
$$

in which we have defined $P=\left[p_{i j}\right]$ and $K \triangleq\left[\begin{array}{lll}k_{1} & k_{2} & k_{3}\end{array}\right]$. From the above two equations and the requirement $P \geqslant 0$, we have

$$
P=\left[\begin{array}{ccc}
0 & 0 & 0 \\
0 & 0 & 0 \\
0 & 0 & p_{33}
\end{array}\right]
$$

where $p_{33}>0$, and $R_{2} k_{1}=0, k_{1}+k_{2} R_{2}=0$. Note that if $R_{2}>0$, then we have $k_{1}=k_{2}=0$. Furthermore, the resulting gain matrix $K$ cannot satisfy (7) for all values of $k_{3}$. Similarly, if $R_{2}=0$, it follows that $k_{1}=0$, so that again (7) cannot be satisfied for all values of $k_{2}$ and $k_{3}$.

\section{Example 4.7}

Finally, we consider a stable linear system with stabilizing linear feedback that when saturated is not globally stabilizing. ${ }^{12}$ Consider (15) where

$$
A=\left[\begin{array}{rrr}
0 & 1 & 0 \\
0 & 0 & 1 \\
-6 & -11 & -6
\end{array}\right], \quad B=\left[\begin{array}{l}
0 \\
0 \\
1
\end{array}\right], \quad K=\left[\begin{array}{lll}
-l^{3}+6 & -3 l^{2}+11 & -3 l+6
\end{array}\right]
$$

Note that the Jordan form of $A+B K$ is

$$
\left[\begin{array}{rrr}
-l & 1 & 0 \\
0 & -l & 1 \\
0 & 0 & -l
\end{array}\right]
$$

which indicates that the unsaturated closed-loop system is globally asymptotically stable for $l>0$. Letting $R=\operatorname{diag}\left[\begin{array}{lll}r_{11} & r_{22} & r_{33}\end{array}\right], P=\left[p_{i j}\right]$ and solving (4), (5) yields

$$
\begin{aligned}
& r_{11}=N(-216 l+432)+R_{2}\left(12 l^{3}-72\right) \\
& r_{22}=N\left(22 l^{3}-36 l^{2}-294 l+588\right)+R_{2}\left(-12 l^{3}+66 l^{2}-36 l-98\right) \\
& r_{33}=N\left(-2 l^{3}+36 l^{2}-150 l+180\right)+R_{2}\left(-6 l^{2}+36 l-50\right)
\end{aligned}
$$


and

$$
\begin{aligned}
& p_{11}=N\left(6 l^{3}-396 l+756\right)+R_{2}\left(11 l^{3}+18 l^{2}-132\right) \\
& p_{12}=N\left(18 l^{2}-216 l+366\right)+R_{2}\left(6 l^{3}+18 l-72\right) \\
& p_{13}=N(-18 l+36)+R_{2}\left(l^{3}-6\right) \\
& p_{22}=N\left(6 l^{3}+33 l^{2}-378 l+599\right)+R_{2}\left(-l^{3}+18 l^{2}+33 l-126\right) \\
& p_{23}=N\left(l^{3}-33 l+60\right)+R_{2}\left(3 l^{2}-11\right) \\
& p_{33}=N\left(3 l^{2}-18 l+25\right)+R_{2}(3 l-6)
\end{aligned}
$$

Solving (6) yields

$$
0<R_{2}+N(3 l-6)
$$

In addition to this, we need also that $R>0$, or equivalently, $r_{11}>0, r_{22}>0, r_{33}>0$. All these conditions constitute the constraint on the parameter $l$. At first, if $N=0$, then it is easy to show that the range of $l$ which provides globally asymptotic stability is $2 \cdot 18<l<3 \cdot 82$. Then, without loss of generality, we choose $N=1$. It can be shown that if $0.93<l<12 \cdot 3$ then there exists $R_{2}$ that satisfies the required conditions and the resulting closed-loop system is globally asymptotically stable in the presence of saturation. This demonstrates that the addition of the integral term to the Lyapunov function used by Theorem 2.1 helps to improve the prediction of the required range of $l$. Note that through numerical experiment, we found that if $x_{0}=[100-100100]^{\mathrm{T}}$, saturation level $\bar{u}=10$, and $0<l \leqslant 28 \cdot 5$, then we have a globally asymptotically stable system. However, if, $l=28.6$ a limit cycle will be observed.

\section{CONCLUSION}

In this paper, we presented a Lyapunov function given in Theorem 2.1 for a system with a saturated controller. This Lyapunov function is composed of a positive semidefinite quadratic term and an integral term. By using this Lyapunov function we showed that a double integrator system can be globally stabilized by a saturating linear controller, whereas, for a triple-integrator system the saturated linear controller does not satisfy the conditions of Theorem 2.1, which agrees with the known result. Several examples are given to illustrate the usage of the main theorem.

\section{APPENDIX}

\section{Notation}

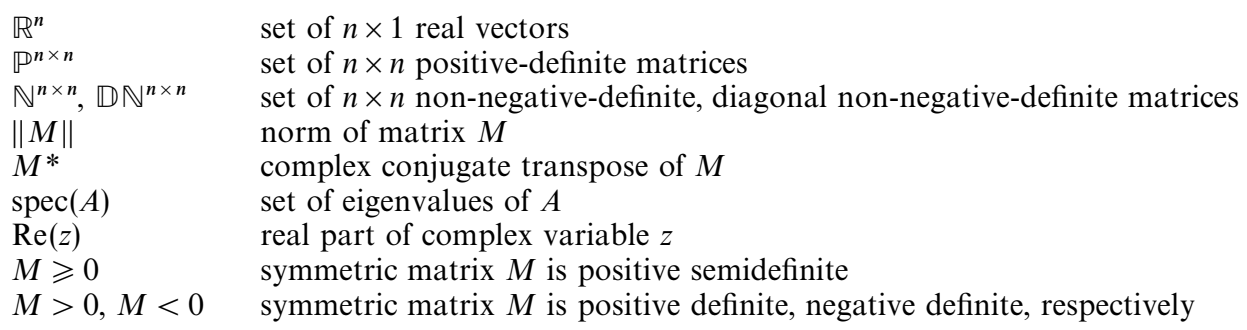




$$
\begin{array}{ll}
Z_{2}, Z_{3}, \ldots & \triangleq\left[\begin{array}{ll}
0 & 1 \\
0 & 0
\end{array}\right],\left[\begin{array}{lll}
0 & 1 & 0 \\
0 & 0 & 1 \\
0 & 0 & 0
\end{array}\right], \cdots \\
J, I_{r} & \triangleq\left[\begin{array}{rr}
0 & 1 \\
-1 & 0
\end{array}\right], r \times r \text { identity matrix }
\end{array}
$$

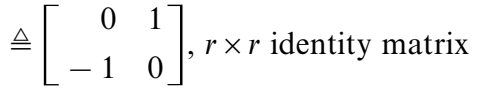

$\operatorname{vec}(\cdot), \otimes, \oplus$

column-stacking operator, Kronecker product and sum

IA, OLHP imaginary axis, open left half-plane

\section{ACKNOWLEDGEMENTS}

This research was supported in part by the National Science Council, Taiwan, under grant NSC-86-2212E-032-010.

\section{REFERENCES}

1. Bernstein, D. S. and A. N. Michel, 'A chronological bibliography on saturating actuators', Int. J. Robust Nonlinear Control, 5, 375-380 (1995).

2. Schmitendorf, W. E. and B. R. Barmish, 'Null controllability of linear systems with constrained controls', SIAM J. Control Optim., 18, 327-345 (1980).

3. Sontag, E. D., 'An algebraic approach to bounded controllability of linear systems', Int. J. Control, 39, 181-188 (1984).

4. Sussmann, H. J., E. D. Sontag and Y. Yang, 'A general result on the stabilization of linear systems using bounded controls', IEEE Trans. Autom. Control, 39, 2411-2425 (1994).

5. Teel, A. R., 'Global stabilization and restricted tracking for multiple integrators with bounded controls', System Control Lett., 18, 165-171 (1992).

6. Narendra, K. S. and J. H. Taylor, Frequency Domain Criteria for Absolute Stability, Academic Press, New York, 1973.

7. Haddad, W. M. and D. S. Bernstein, 'Parameter-dependent Lyapunov functions and the Popov criterion in robust analysis and synthesis', IEEE Trans. Automat. Control, AC-40, 536-543 (1995).

8. Fuller, A. T., 'In-the-large stability of relay and saturating control systems with linear controllers', Int. J. Control, 10, 457-480 (1969).

9. Chen, C. T., Linear System Theory and Design, Rinehart and Winston, New York, 1984.

10. Bekker, P. A., 'The positive semidefinteness of partitioned matrices', Linear Algebra Appl., 261-278 (1998).

11. Sirisena, H. R., 'Optimal control of saturating linear plants for quadratic performance indices', Int. J. Control, 8(1), 65-87 (1978).

12. Aguirre, B. et al., 'First harmonic analysis of linear control systems with high-gain saturating feedback', Int. J. Bifurcation Chaos, 7(11), 2501-2510 (1997). 